\title{
Utilization of arterial blood gas measurements in a tertiary care hospital
}

\begin{abstract}
Context: There has been very few publications answering why the Arterial Blood Gas (ABG) test being ordered and whether its results affected patient management. Dissemination of guidelines, educational efforts can help in decreasing the unnecessary utilization of ABG thereby reducing the costs and complication.

Aims: To describe the pattern of Arterial Blood Gas utilization in a tertiary intensive care unit.

Settings and Design: This prospective observational study was conducted in the adult ICU consisting of 11 beds.

Methods and Material: About $486 \mathrm{ABG}$ tests were performed and analyzed during three months duration. The clinician filled out ABG utilization proforma after test was done.

Results: The common reasons for requesting an $\mathrm{ABG}$ test were routine (60.28\%), following respiratory events $(11.72 \%)$, and follow up on abnormal reports $(9.25 \%)$. The clinicians considered $\mathrm{PO} 2, \mathrm{pH}$, and $\mathrm{PCO} 2$ the most important parameters in the $\mathrm{ABG}$ test results. Of the results, there was no intervention in $52.2 \%$ cases and ventilator settings were changed only in $29.8 \%$ of cases.
\end{abstract}

Conclusion: Oftentimes, many ABG tests were ordered as part of a routine investigation. ABG Analysis was done without test results influencing patient management.

Keywords: arterial blood gas, intensive care unit, utilization
Volume 6 Issue 2 - 2016

Sushil Khanal

Grande International Hospital, Nepal

Correspondence: Sushil Khanal, Grande International Hospital, Dhapasi, Kathmandu, Nepal,Tel +977-9843I77465, Email khanaliom@gmail.com

Received: June 06,2016 | Published: November 09, 2016

\section{Introduction}

The arterial blood gas $(\mathrm{ABG})$ is a frequently ordered laboratory test. Laboratory tests should be ordered to make accurate diagnoses, change management, monitor the response to management. ${ }^{1,2}$ The continued frequency of $\mathrm{ABG}$ valuation may be influenced by routine work-patterns and easy access via arterial lines. ${ }^{3}$ Many laboratory analysis including $\mathrm{ABG}$ are repeated too early. ${ }^{4}$ Inappropriate utilization and blood collection not only escalate cost but may also create unnecessary patient discomfort and complications. ${ }^{5}$ Ancillary expenditures in the intensive care setting can be decreased without compromising care of the patient. ${ }^{6}$ Implementation of practice guidelines for blood gas measurement has been shown to decrease test utilization without affecting outcome. ${ }^{?}$

We decided to examine $\mathrm{ABG}$ test utilization patterns in our Intensive Care Unit (ICU) in an attempt to identify opportunities for improved utilization. We studied why the ABG test being ordered and whether its results affected patient management.

\section{Subjects and methods}

The ethical approval was taken from ethical committee of the hospital. This prospective observational study was conducted in the adult ICU consisting of 11 beds. ABG tests performed outside ICU were not included in this study. The blood gas analyzers are validated to report arterial $\mathrm{pH}, \mathrm{PCO} 2, \mathrm{PO} 2$, oxygen saturation, hemoglobin, hematocrit, sodium, potassium, ionized calcium, and whole blood glucose values.

The clinician (doctor or nurse) filled out the ABG Survey form after ABG test was done. The utilization survey inquired about the reason for ordering the $\mathrm{ABG}$ test, the most important parameters used, and the effect of the results on patient management. The data collection took approximately three months (June to August 2015).

\section{Results}

We collected and analyzed 486 ABG test results during the study period. The common reasons for requesting an $\mathrm{ABG}$ test were routine $(60.28 \%)$ following respiratory events $(11.72 \%)$ and follow up on abnormal reports $(9.25 \%)$ (Table 1). The clinicians considered PCO2, $\mathrm{pH}, \mathrm{PO} 2$, the most important parameters in $\mathrm{ABG}$ test results (Table 2). Of the results there was no intervention in $52.2 \%$ cases and ventilators setting were changed only in $29.8 \%$ of cases (Table 3 ).

Table I Reason for performing arterial blood gas measurement in 486 cases

\begin{tabular}{ll}
\hline & No.* (\%) of cases \\
\hline Routine & $293(60.28)$ \\
Respiratory event & $57(\mathrm{II} .72)$ \\
Follow-up on abnormal results & $45(9.25)$ \\
Change ventilator setting & $41(8.43)$ \\
Metabolic event & $23(4.73)$ \\
Postextubation & $18(3.70)$ \\
Preintubation & $15(3.08)$ \\
Preextubation & $12(2.46)$ \\
Cardiac event & $9(1.85)$ \\
Unreliable pulse oximeter data & $8(1.64)$ \\
Postintubation & $6(1.23)$ \\
Postprocedure laboratory testing & $5(1.02)$ \\
Low hematocrit value & $3(0.6)$ \\
Altered mental status & $1(0.2)$
\end{tabular}

*Clinicians were allowed to mark more than I reason for ordering the test. 
Table 2 Parameters considered most important by clinicians

\begin{tabular}{ll}
\hline & No** (\%) of cases \\
\hline $\mathrm{PCO}_{2}$ & $456(93.82)$ \\
$\mathrm{pH}$ & $453(93.2)$ \\
$\mathrm{PO}_{2}$ & $442(90.9)$ \\
$\mathrm{HCO}_{3}$ & $297(6 \mathrm{I} .1)$ \\
Lactate & $295(60.6)$ \\
Oxygen saturation & $29 \mathrm{I}(59.8)$ \\
Electrolyte & $173(35.5)$ \\
Hematocrit & $\mathrm{I} 28(26.3)$ \\
\hline
\end{tabular}

*Clinicians were allowed to mark more than one parameter.

Table 3 Change in management in response to arterial blood gas test results

\begin{tabular}{ll}
\hline & No.* (\%) of cases \\
\hline No Interventions & $254(52.2)$ \\
Change ventilator settings & $168(34.5)$ \\
Intubate & $15(3.08)$ \\
Extubate & $12(2.46)$ \\
Electrolyte Correction & $10(2.05)$ \\
Dialysis & $5(1.02)$ \\
\hline
\end{tabular}

*Clinicians were allowed to mark more than one parameter.

\section{Discussion}

Our study reveals that many ABGs were just done routinely without influencing the patients' management. This result can be helpful in developing a way that can decrease the number and cost of ABG utilization without compromising the quality of care. Various interventions like education, guideline management and computer model have been practiced to reduce the laboratory utilization. Wang et al. ${ }^{8}$ demonstrated the modest reduction in routine testing after development of guideline, order template design and education. Computer based model reminding the physician about the redundant laboratory test has been effective to some extent to reduce the number of test performed. ${ }^{9}$ Combination of different intervention model can decrease the unnecessary sampling of blood through arterial catheter. Our some clinical practice pattern are often based on expert opinion, historical practice and blind acceptance rather on evidence base. ${ }^{10}$ Some results of ABG analysis do not benefit the patients but they can make physicians feel more comfortable in knowing how is the patient. Unless the intervention is free of cost and carries no potential risks, it is a slippery slope to justify it is based on comfort and convenience of caregivers. ${ }^{11}$

The indications of arterial blood gas analysis are not specific as the indication for placement of arterial catheter. Some of the indications for arterial blood gas analysis which are being described in literature have been questioned by some of the studies. Recently two studies examining the usefulness of $\mathrm{ABG}$ tests in spontaneous breathing trial to predict successful extubation concluded that $A B G$ values affected extubation decisions in relatively few patients. ${ }^{12,13}$ Despite advances in non invasive monitoring in critical care, invasive techniques still being practiced in critically ill patients. Pulse oximetry could replace for arterial blood gas analysis in some circumstances. The use of the $\mathrm{SpO} 2 / \mathrm{FIO} 2$ ratio may better facilitate the screening and rapid identification of patients with lung injury while avoiding the blood use and cost for blood gas determinations. ${ }^{14}$

It is our impression that ICU patients continue to have arterial catheter as it is easy to draw blood from the catheter. Presence of arterial catheter has been a sole factor in unnecessary utilization of arterial blood test. ${ }^{15}$ Not only this increases the ICU cost but also direct and indirect complications associated with arterial catheterization. At our institution, charge for an ABG costs $\$ 10$. From the study we estimated that $50 \%$ of $\mathrm{ABG}$ tests performed were unnecessary. This would cost around $\$ 10000$ annually excluding the cost of reagents.

It is important to obtain data quickly so as to optimize the therapeutic response interval and allow prompt treatment in ICU. The use of central laboratory testing in a hospital may cause a long delay between the time at which a test is ordered and the time at which the result is received by a clinician; such delays may compromise the treatment of critically ill patients. ${ }^{16}$ If analysis like ABG is performed in the ICU several processing steps can be eliminated, results are obtained rapidly, patient management is timely, and outcomes improve. This could be one of the reasons behind the rising use of ABG in ICU. Blum et al. ${ }^{17}$ suggest that a significant reduction in $\mathrm{ABG}$ analysis is safe and does not negatively impact patient outcome, and it may reduce the likelihood of potential complications from arterial puncture. In an era of rising medical costs and increasing patient complexity, judicious and meaningful use of invasive medical resources like $\mathrm{ABG}$ has become an increasingly important part of the healthcare delivery discussion. ${ }^{18}$

There are several limitations of this study. As this is an observational study, we only calculated the number of $\mathrm{ABG}$ tests that were ordered unnecessarily, and we did not determine whether not performing an ABG test in these situations would affect the patient's outcome. We used a survey to obtain information on ABG test utilization. Surveys have limitations, including interpretation and recall bias. Despite these limitations, the results of this preliminary study are promising. The appropriate justification of any laboratory intervention is proved when the results are beneficial to the care of the patients. Every intervention has real costs and real risks, interventions without benefits should not be routinely used. Dissemination of guideline, educational efforts and reminding of redundant $\mathrm{ABG}$ analysis can help in decreasing the unnecessary utilization of $A B G$ thereby reducing the cost and complications. Multiple randomized studies in different patients subgroups will be needed to clarify whether arterial gas analysis influences the outcome.

\section{Conflicts of interest}

The authors declare there are no conflicts of interest.

\section{Acknowledgments}

None.

\section{Funding}

None.

\section{References}

1. Lundberg GD. The need for an outcomes research agenda for clinical laboratory testing. JAMA. 1998;280(6):565-566.

2. Van Walraven C, Naylor CD. Do we know what inappropriate laboratory utilization is? A systematic review of laboratory clinical audits. JAMA. 1998;280(6):550-558

3. Roberts D, Ostryzniuk P, Loewen E, et al. Control of blood gas measurements in the intensive care units. Lancet. 1991;337(8757):1580 1582 .

4. Bates DW, Boyle DL, Rittenberg E, et al. What proportion of common diagnostic tests appears redundant? Am J Med. 1998;104(4):361-368. 
5. Melanson SE, Szymanski T, Rogers SO, et al. Utilization of arterial blood gas measurements in a large tertiary care hospital. Am J Clin Pathol. 2007;127(4):604-609.

6. Bansal P, Aronsky D, Aronsky D, et al. A computer based intervention on the appropriate use of arterial blood gas. Proc AMIA Symp. 2001:32-36.

7. Pilon CS, Leathley M, London R, et al. Practice Guidelines for arterial blood gas measurement in the intensive care unit decreases number and increases appropriateness of tests. Crit Care Med. 1997;25(8):13081313 .

8. Wang TJ, Mort EA, Nordberg $\mathrm{P}$, et al. A utilization managemen intervention to reduce unnecessary testing in the coronary care unit. Arch Intern Med. 2002;162(16):1885-1890.

9. Bates DW, Kuperman GJ, Rittenberg E, et al. A randomized trial of a computer-based intervention to reduce utilization of redundant laboratory tests. Am J Med. 1999;106(2):144-150.

10. Singer M, Glynne P. Treating critical illness: the importance of first doing harm. PLoS Med. 2005;2(6):e167.

11. Garland A. Arterial lines in the ICU: a call for rigorous controlled trials Chest. 2014;146(5):1155-1158.
12. Pawson SR, DePriest JL. Are blood gases necessary in mechanically ventilated patients who have successfully completed a spontaneous breathing trial? Respir Care. 2004;49(11):1316-1319.

13. Salam A, Smina M, Gada P, et al. The effect of arterial blood gas values on extubation decisions. Respir Care. 2003;48(11):1033-1037.

14. Rice TW, Wheeler AP, Bernard GR, et al. Comparison of the SpO2/ $\mathrm{FIO} 2$ ratio and the $\mathrm{PaO} 2 / \mathrm{FIO} 2$ ratio in patients with acute lung injury or ARDS. Chest. 2007;132(2):410-417.

15. Browning JA, Kaiser DL, Durbin CG. The effect of guidelines on the appropriate use of arterial blood gas analysis in the intensive care unit. Respiratory Care. 1989;34:269-276.

16. Kost GJ, Ihrmeyer SS, Chernow B, et al. The laboratory- clinical interface: Point-of-care testing. Chest. 1999;115(4):1140-1154.

17. Blum FE, Lund ET, Hall HA, et al. Reevaluation of the utilization of arterial blood gas analysis in the Intensive Care Unit: effects on patient safety and patient outcome. J Crit Care. 2015;30(2):438.e1-e5.

18. DellaVolpe JD, Chakraborti C, Cerreta K, et al. Effects of implementing a protocol for arterial blood gas use on ordering practices and diagnostic yield. Healthc (Amst). 2014;2(2):130-135. 